\title{
Supporting information for InP Nanoflag Growth from a Nanowire Template by in Situ Catalyst Manipulation
}

Alexander Kelrich, ${ }^{*}{ }^{\dagger}$ Ofir Sorias, ${ }^{\dagger}$ Yonatan Calahorra,${ }^{\dagger} \S$ Yaron Kauffmann,${ }^{\dagger}$ Ran Gladstone, ${ }^{\dagger}$ Shimon Cohen,$^{\dagger}$ Meir Orenstein, ${ }^{\dagger}$ and Dan Ritter ${ }^{*}{ }^{\dagger}$

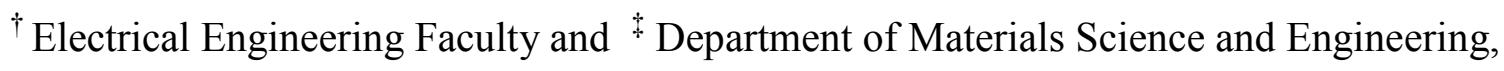
Technion - Israel Institute of Technology, Haifa 32000, Israel

$\S^{\S}$ Department of Materials Science and Metallurgy, University of Cambridge, Cambridge CB3 0FS, United Kingdom

*E-mail: skelrich@campus.technion.ac.il,ritter@ee.technion.ac.il 


\section{Contents}

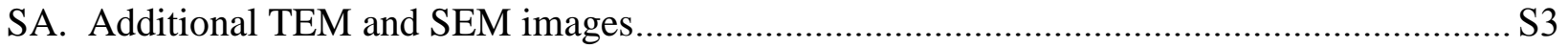

SB. Micro-PL setup used for optical characterization .......................................................... S6

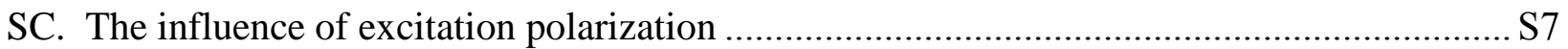

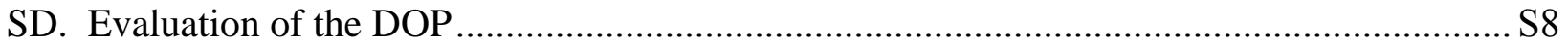




\section{SA. Additional TEM and SEM images}

Figure S1 shows additional HR-TEM images of oblique facets created during catalyst unpinning. Multiple oblique facets on the same tip prevailed in nanowires with a diameter above $60 \mathrm{~nm}$.
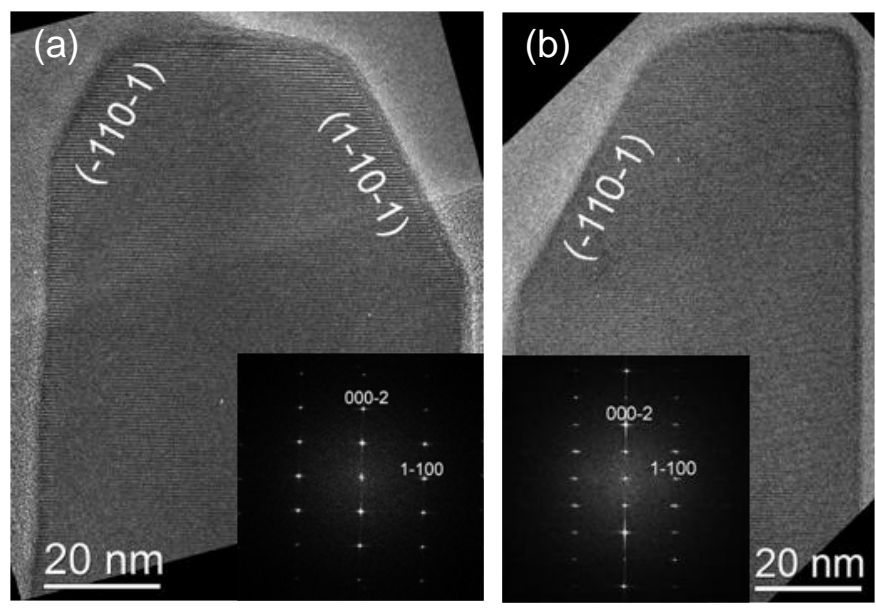

Figure S1. Truncated area of InP nanowires following unpinning procedure imaged at $\langle 11-20\rangle$ zone axis. (a) $70 \mathrm{~nm}$ wide nanowire with more than one truncated facet. (b) Single truncation in $62 \mathrm{~nm}$ wide nanowire. The insets show FFT patterns illustrating the pure WZ structure. Not a single stacking fault is observed.

Figure S2 shows SEM top-view of nanostructures, exhibiting the case of catalyst mediated radial growth on top of three side facets, rather than catalyst migration along the nanowire axis during the catalyst unpinning step.
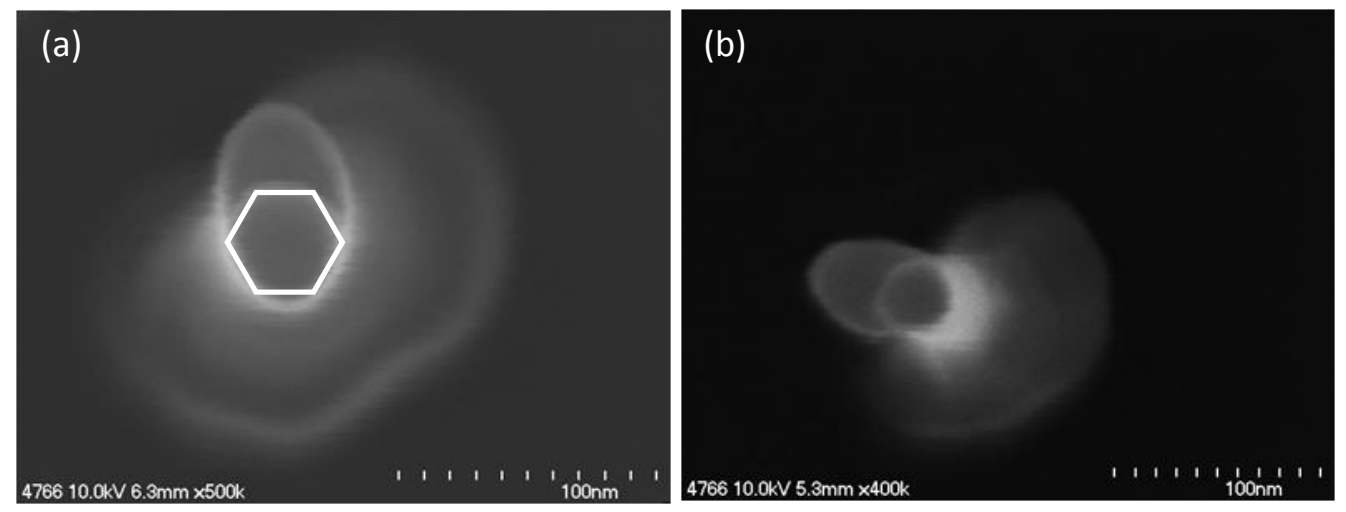

Figure S2. Top-view SEM images of InP nanowiress after catalyst unpinning procedure demonstrating radial growth initiation. The growth takes place on three side-facets and its thickness is equal to the nanowire diameter at the growth nucleation point (the nanowire is slightly tapered). Hexagonal cross-section of the primary nanowire is indicated in (a). 
Figure S3 was used for evaluation of growth direction distribution of the nanoflags, summarized in Figure 3c. Only successfully grown flag-like structures at $\sim 95 \%$ yield were taken into consideration.

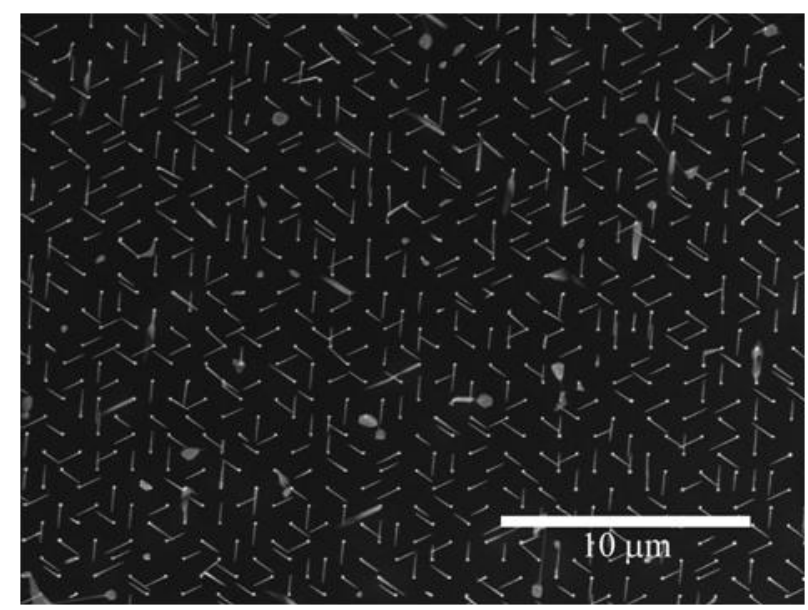

Figure S3. Top view SEM image of InP nanoflag array comprising $\sim 700$ properly grown flags at $95 \%$ yield.

Figure S4 demonstrates that the nanoflag thickness is limited only by primary nanowire diameter. $\sim 20 \mathrm{~nm}$ nanoflags are grown on $\sim 6 \mu \mathrm{m}$ long nanowire "flagpoles".

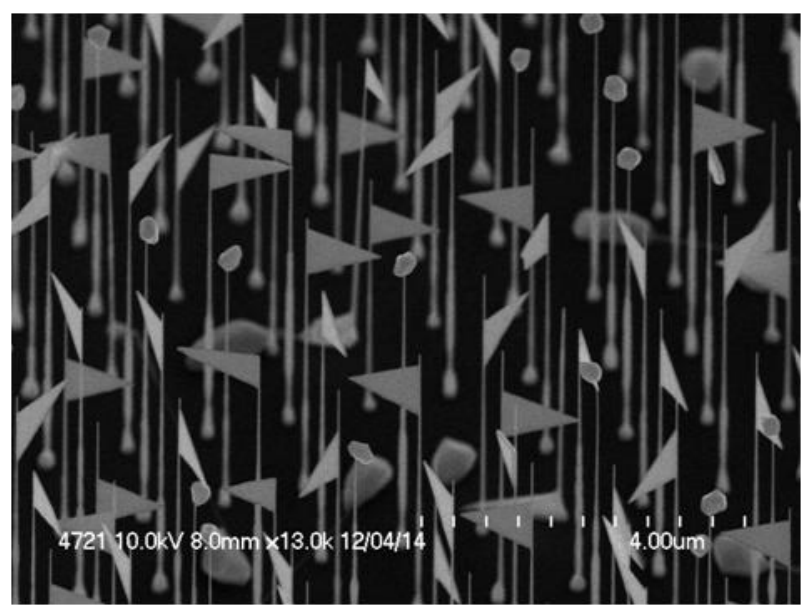

Figure S4. SEM image of nanoflag array tilted at $30^{\circ} . \sim 20 \mathrm{~nm}$ nanoflags are grown on $\sim 6 \mu \mathrm{m}$ long nanowire "flagpoles". The bulges at the top of some of the nanowires are created due to incomplete unpinning followed by simultaneous growth at various directions. 
Implementing identical growth procedure as described in stages II-VI in the main text, but without interruption of TMI flow during the annealing stage, results in unpinned catalyst leading to symmetric radial growth on all six sidewalls near the nanowire tip. The catalyst acts as effective precursor sink but can't guide growth according to specific direction, since no sidewall transition occurred, resulting in uniform shell shown in Figure S5.

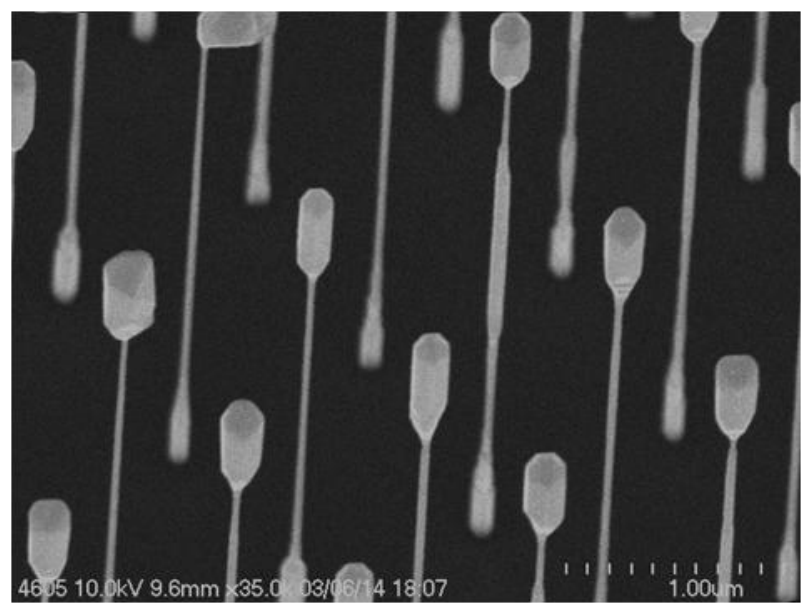

Figure S5. SEM image of nanowire array tilted at $30^{\circ}$. Following the nanowire growth, the temperature was gradually increased from $420^{\circ} \mathrm{C}$ to $515^{\circ} \mathrm{C}$ without stopping the TMI flow; growth continued for 40 minutes. Symmetric growth on all six sidewalls near the nanowire tip takes place, since the catalyst is pinned on (0001) facet and acts as effective precursor sink but can't guide growth according specific direction. 
Figure S6 shows HR-TEM image of the transition area from high defect density segment to pure WZ phase nanowire structure. The change of crystal structure is achieved solely by phosphorus flow adjustment.

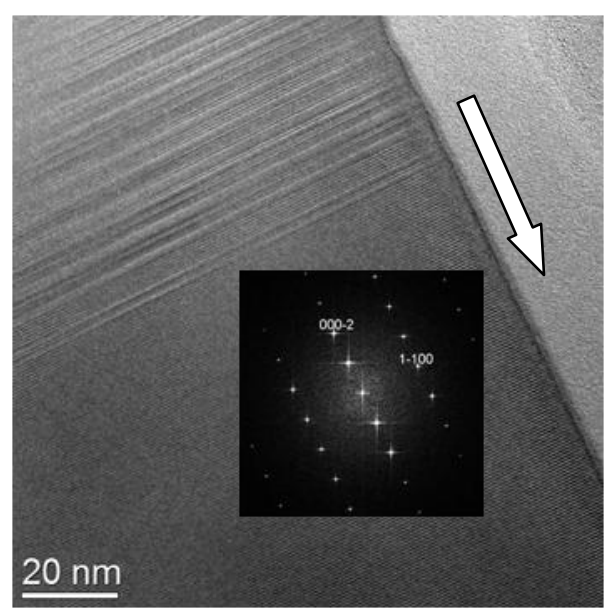

Figure S6 HR-TEM image of middle part of the nanoflag containing high defect density segment, created for catalyst pinning. The inset shows FFT indicating pure WZ structure of the nanowire. The arrow indicates growth direction of the nanowire.

\section{SB. Micro-PL setup used for optical characterization}

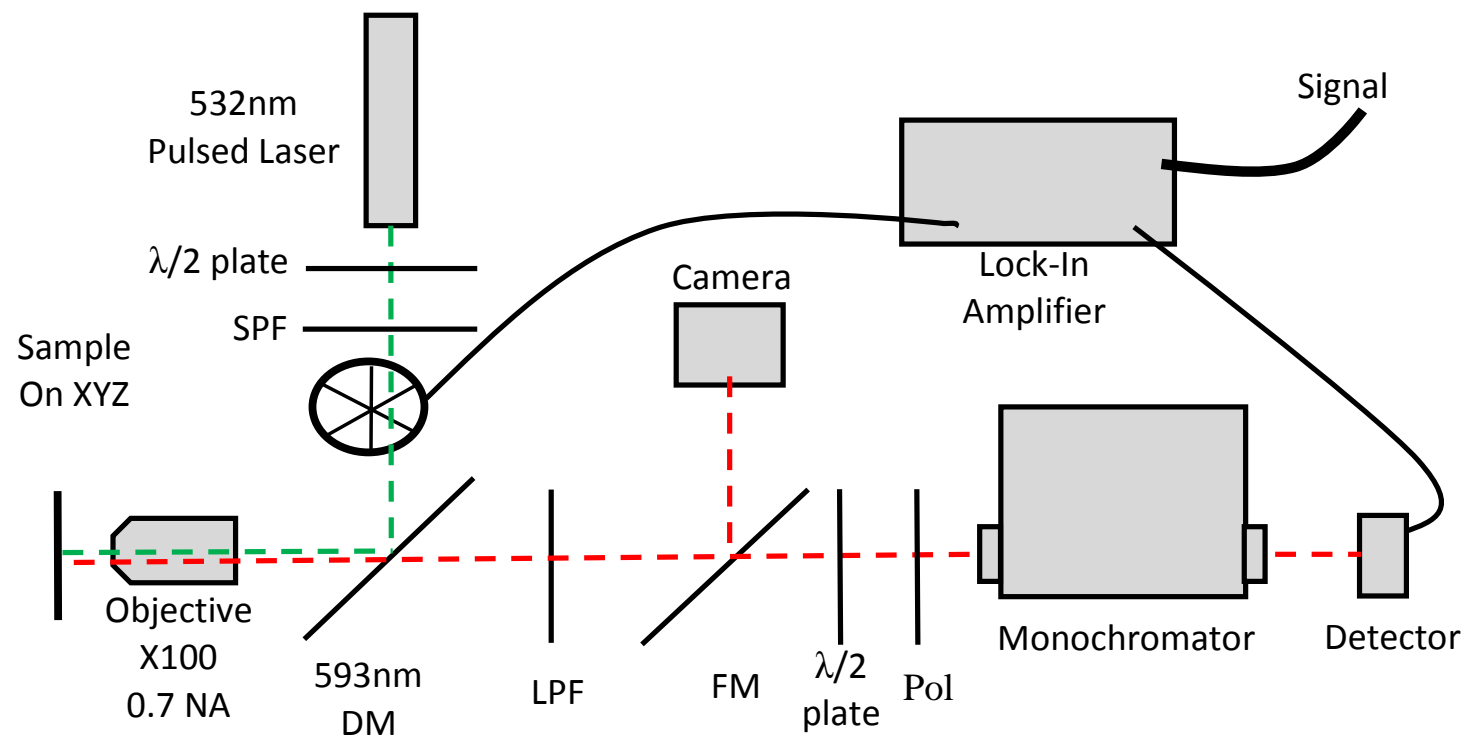

Figure S7. Micro-PL setup used for nanoflag characterization. 


\section{SC. The influence of excitation polarization}

Laser interaction with a nanoflag was evaluated by FDTD simulations. The scattering cross-section shown in Figure S8 is an indication of this interaction. The laser interacts strongly with the flag if its polarization is parralell to the flag direction (defined in the simulation as 90 degrees polarization). Up to a deviation of 30 degrees the intercation is still significant. When the polarization is perpendicular to the flag direction, the intercation is very weak, due to the small width of the flag. At resonance, strong interaction of the exciting light with the nanoflag takes place.

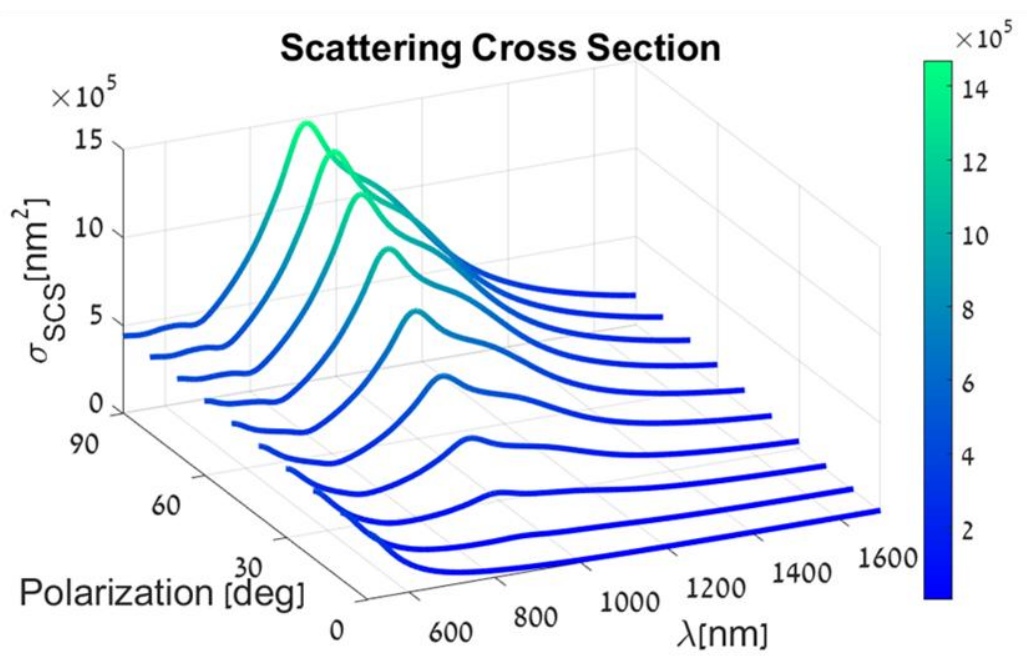

Figure S8. Simulations of a typical scattering cross section from a nanoflag with respect to the laser polarization. At 90 degrees the laser polarization is parallel to the nanoflag elongation axis. 
Figure S9 demonstrates the PL intensity dependence of a nanoflag array on the exciting laser polarization, as recorded by a camera. Figures S9, panels a-c show the recorded PL for different laser polarizations indicated by the arrows. Figure S9, panel d shows SEM picture of the same array area emphasizing that flag orientations coincide with the laser polarization for efficient excitation of non-resonant transitions due to the nanoflag geometry.
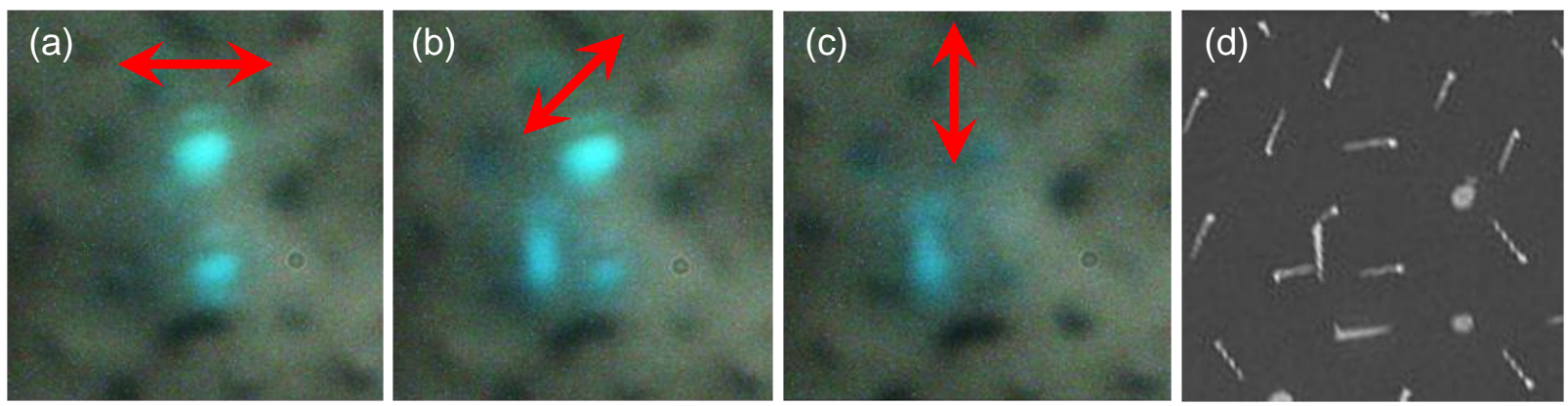

Figure S9. (a)-(c) Camera pictures showing PL dependence on input laser polarization, indicated by red arrows. (d) SEM image of the array shown in (a)-(c). The inter-flag distance is $2 \mu \mathrm{m}$.

\section{SD. Evaluation of the DOP}

Analysis of 15 polarization resolved PL measurements of different nanoflags at $860 \mathrm{~nm}$, shows that the polarization angles are $20^{\circ}, 80^{\circ}$ or $140^{\circ}$, with DOP in the range of $60 \%-70 \%$, $80 \%-88 \%, 42 \%-50 \%$, respectively. The variance for each polarization angle can be explained by relatively low signal-to-noise ratio and weak contributions from differently oriented neighboring nanoflags and isotropic substrate PL. However, the distinct difference in the DOP between different polarization angles was found to be due to the measurement setup, and mostly due to phase shift caused by the dichroic mirror, resulting in drastic reduction of the measured DOP for diagonal polarizations. To overcome this, system response was measured by inserting 1:10000 polarized light at 860nm, and used as normalization for the results shown in Figure 6, panels c-e. After normalization the DOP is in the range of $70 \%-90 \%$. 